Document downloaded from:

http://hdl.handle.net/10251/53514

This paper must be cited as:

Sotelo Orozco, A.; Guijarro Estelles, ED.; Trujillo, L. (2015). Seizure states identification in experimental epilepsy using gabor atom analysis. Journal of Neuroscience Methods. 241:121-131. doi:10.1016/j.jneumeth.2014.12.001.

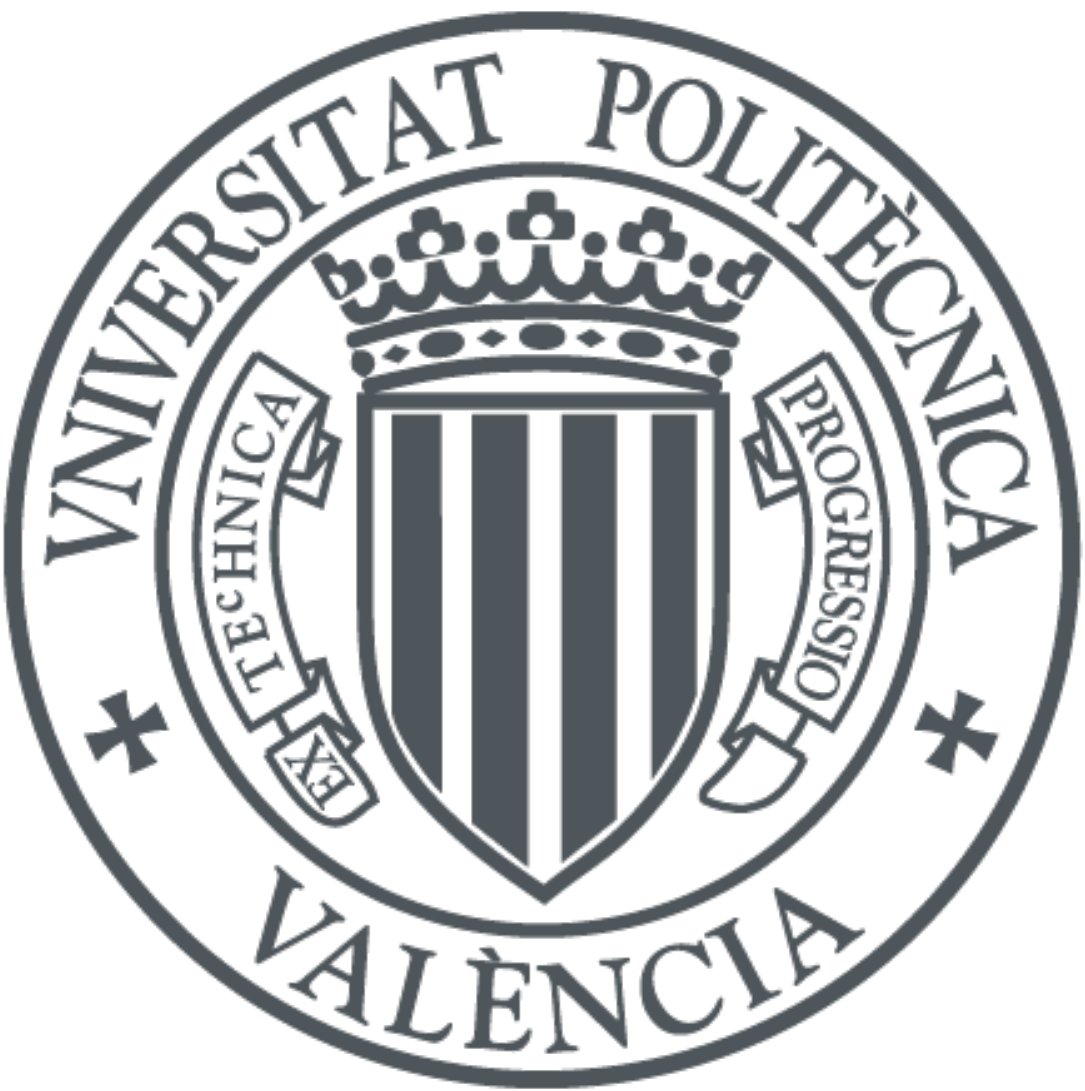

The final publication is available at

http://dx.doi.org/10.1016/j.jneumeth.2014.12.001

Copyright Elsevier

Additional Information 


\title{
Seizure States Identification in Experimental Epilepsy using Gabor Atom Analysis
}

\author{
Arturo Sotelo $^{\mathrm{a}, \mathrm{b}, *}$, Enrique D. Guijarro ${ }^{\mathrm{b}}$, Leonardo Trujillo ${ }^{\mathrm{a}}$ \\ ${ }^{a}$ Instituto Tecnológico de Tijuana. Blvd. Industrial S/N. Tijuana B.C., México. Tel.: \\ $+52(664) 6244743$ \\ ${ }^{b}$ Universitat Politécnica de Valéncia, Cami de Vera S/N, 46022 Valencia,Spain, Tel: 00 \\ 34963877000 (Ext.:76065)
}

\begin{abstract}
Background:

Epileptic seizures evolve through several states, and in the process the brain signals may change dramatically. Signals from different states share similar features, making it difficult to distinguish them from a time series; the goal of this work is to build a classifier capable of identifying seizure states based on time-frequency features taken from short signal segments.
\end{abstract}

\section{Methods:}

There are different amounts of frequency components within each Time-Frequency window for each seizure state, referred to as the Gabor atom density. Taking short signal segments from the different states and decomposing them into their atoms, the present paper suggests that is possible to identify each seizure state based on the Gabor atom density. The brain

${ }^{*}$ Corresponding author

Email addresses: asotelo@tectijuana.mx (Arturo Sotelo), eguijarro@eln.upv.es (Enrique D. Guijarro), leonardo.trujillo@tectijuana.edu.mx (Leonardo Trujillo) 
signals used in this work were taken form a database of intracranial recorded seizures from the Kindling model.

Results:

The findings suggest that short signal segments have enough information to be used to derive a classifier able to identify the seizure states with reasonable confidence, particularly when used with seizures from the same subject. Achieving average sensitivity values between 0.82 and 0.97 , and area under the curve values between 0.5 and 0.9 .

\section{Conclusions:}

The experimental results suggest that seizure states can be revealed by the Gabor atom density; and combining this feature with the epoch's energy produces an improved classifier. These results are comparable with the recently published on state identification. In addition, considering that the order of seizure states is unlikely to change, these results are promising for automatic seizure state classification.

Keywords: Epilepsy, Seizure states, ECoG, Kindling model, Matching Pursuit, Gabor atoms density.

\section{Introduction}

There are several neurological disorders that affect the human brain, one of the most serious and common is epilepsy (Witee et al., 2003). According to different studies, the number of persons with epilepsy varies depending on the region and the considered population. In studies from The World Health 
Organization (WHO, 2005), the estimated mean of people with epilepsy per 1000 is 8.93. An epileptic seizure may affect the brain partially or completely, producing partial or generalized seizures respectively (Morimoto et al., 2004). Seizures will manifest in the electrical activity produced by the brain. Placing electrodes directly inside the brain or over the cerebral cortex allows the recording free from artifacts (Zaveri et al., 1992), that are called Electrocorticograms $(E C o G)$.

Epileptic seizures are dynamic processes evolving throughout four main states (Franaszczuk et al., 1998; Iasemidis et al., 2003); those states are: (1) the Basal state (2) the Pre-Ictal state, (3) the Ictal state; and (4) the Post-Ictal state. Within the time-domain the ECoG shows amplitude changes while an epileptic seizure episode is in progress, and the signal's morphology is different at each state (Cockerell, 2003). In general, ECoG could be affected by the state of awareness of the subject. When the brain functions are normal it is considered the Basal state, the ECoG is characterized by a low amplitude and relative high frequency. In the Pre-Ictal state, the corresponding ECoG shows an amplitude increase with respect to the Basal state. There are spikes and transitory activity but no definitive evolution, known as recruiting rhythms (Kohsaka et al., 2002; Roso and Figliola, 2004). Though, during this state the individual may not exhibit clinical manifestations. The Ictal state is precisely when the individual exhibits more evident clinical manifestations of the seizure; in cases when these discharges become widespread enough it might resulting in a convulsive response (Morimoto et al., 2004). The $E C o G$ during this state is characterized by high amplitude discharges, a low frequency and a predominant rhythmicity. The last state is the Post- 
-Ictal, where the ECoG shows general amplitude depression and frequency increases, in this state it is possible to find spike-and-wave complexes; the amplitude keeps decreasing as the $E C o G$ gradually returns to the Basal state.

Epileptic seizures are spontaneous and sometimes are triggered by an external phenomena, mostly happening without any warning, making it hard to study them in humans (Curtis and Avanzini, 2001). Therefore, elicited seizures are used for research purposes by means of animal models, mainly rodents. The epilepsy conditions are achieved in previously healthy (nonepileptic) animals as a result of applying short duration electrical stimulus to the brain, known as the Kindling model(Goddard, 1983; McIntyre and Gilby, 2009); which can produce seizures with precise focal activation (Morimoto et al., 2004).

The seizures elicited by the kindling model are rated according to the subject's clinical manifestation into a five level-scale, known as Racine scale (Racine, 1972). The less severe seizures are considered as focal and they are rated as stage one, as the abnormal discharges become widespread over the brain the clinical manifestations change. When the afterdischarge is capable of stimulating the nearby neurons reaching the cortex, it produces a generalize motor seizure, or stage five, the highest in the Racine scale.

The brain, like most physiological systems, produces signals which posses statistics that vary with time; i.e, they are non-stationary signals (Williams et al., 1995). Fortunately, the rate at which such systems can change is bounded; this allows breaking signals into segments of short fixed duration over which the statistics of interest may be assumed stationary. However, the signal may change within the duration of the window (Rangayyan, 
2002). Therefore a method able to analyze signals with no apparent stationarity is required, in this paper we use the adaptive decomposition algorithm developed by Mallat and Zhang (1993) called Matching-Pursuit algorithm $(M Z M P)$. This algorithm decompose signals in terms of a function dictionary, primitive components called atoms that can provides information about the rhythmic and transient brain activity (Jouny et al., 2003).

The study of the seizure states is motivated by the interest in evaluating experimental epilepsy treatments. However, as a first step, this work focuses on identifying three of the main seizure states: Pre-Ictal, Ictal, and Post-Ictal as it is done in (Sotelo et al., 2007, 2012, 2013). For instance, this makes it possible to estimate when the Ictal state develops. Our approach is to compute the dynamics of short ECoG segments, acquired from elicited seizures using the Kindling model on Wistar rats. The signal dynamics are estimated by the number of frequency components found in the $E C o G$ segment, using a derived measured from the $M Z M P$ designated as Gabor Atom Density $(G A D)$ (Jouny et al., 2003). This numerical feature can be used to monitor changes in ECoG complexity (Jouny et al., 2004), which allows us to perform classification of the seizure states.

Over the past years, many researchers have attempted to develop algorithms for automatic analysis of the $E E G$ with the purpose of identifying or predicting epileptic seizure activity. Using different approaches to analyze features, such as the short-term maximum Lyapunov exponent to reveal the dynamic characteristics of the $E C o G$ during the seizure evolution (Nair et al., 2009). Niknazar et al. (2013) propose a unified thresholding approach using several features from time domain, frequency domain and non-linear 
properties, able to discriminate during seizure and after seizure states. LópezCuevas et al. (2013) propose an algorithm based on artificial neural networks for automatic detection of high frequency oscillations related to epilepsy. Some other uses a technique based on reservoir computing; for instance, Buteneers et al. (2013) propose real-time seizure detection from ECoG and trigger the treatment on a rat model, achieving an average error rate of 2.8\%; and Fu et al. (2014) propose to identify seizure non-seizure activity in humans, reaching a classification accuracy of $99.125 \%$.

\section{Materials and Methods}

The signals data-sets were obtained from a database of the Centro de investigación del Hospital General Universitario de Valencia, working in conjunction with the Universidad Politécnica de Valencia, with the purpose of studding states in elicited seizures. The kindling procedure was carried out in compliance with current European directives for animal experimentation (86/609/ECC) and with those set by the Valencian Community Government, in accordance with the corresponding institutional animal care committee. The procedure was performed as described in (Gallego et al., 2010), using adult subjects weighing 270-310g stereotactically implanted with a bipolar electrode made of twisted pair of Teflon-coated $0.25 \mathrm{~mm}$ diameter stainless steel wires separated by $0.5 \mathrm{~mm}$ at the tip and $8 \mathrm{~mm}$ in length, implanted at the left piriform cortex for the stimulation and recording purpose. These were placed at the coordinates: $0.8 \mathrm{~mm}$ posterior, $4.9 \mathrm{~mm}$ left, and $8.8 \mathrm{~mm}$ ventral from the bregma. With the purpose of signal recording and fixing the connector to the skull, three more electrodes were implanted, two of them 
$1 \mathrm{~mm}$ anterior to the coronal suture, $3 \mathrm{~mm}$ from the mid-line on both sides and one occipital and the third $1 \mathrm{~mm}$ posterior to the lambdoid suture, $3 \mathrm{~mm}$ right from the midline, allowing deep and frontal right signal recording. The standard kindling procedure started 10 days after the surgical procedure, the stimuli was applied on a daily basis consisting of a 1 s train from a $50 \mathrm{~Hz}$ rectangular signal with a $5 \%$ duty-cycle and $500 \mu \mathrm{A}$ intensity, until more than three consecutive seizures of stage 5 were provoked. At this point, the subject was considered fully kindled. Then the seizure threshold (ST) is determined, this is achieved using a stepwise ascent method (Freeman and Jarvis, 1981), consisting on a $50 \mathrm{~Hz}$ rectangular signal with a $5 \%$ duty-cycle by $1 \mathrm{~ms}$, separated by 1 minute intervals. The current intensity starts at $7 \mu \mathrm{A}$, increasing gradually by $20 \%$ at each step until a behavioral seizure occurred or up to the maximum intensity of $500 \mu \mathrm{A}$. The intensity reached when a seizure is produced is considered to be the seizure threshold. After ST is determined, the recording process starts; the $E C o G$ from elicited seizures were recorded from the stimulation and frontal left electrodes, using the frontal right electrode as reference. Eliciting one seizure a day for 9 continuous days. For the recording process, the intensity of the stimulation starts $4 \%$ below the ST intensity, rounding to the nearest integer, increasing $2 \%$ (rounding to the nearest integer) leaving one minute apart among stimulations until a seizure stage 5 is achieved. Before achieving the desired seizure stage, and after every new stimulation the presence of high amplitude wave-spike complexes in $E C o G$ increase. In this work, we consider that this $E C o G$ 's morphology and behavior coincide with the Pre-Ictal state. Therefore, ECoG previous to the ictal state were classified and used as the Pre-Ictal state. 


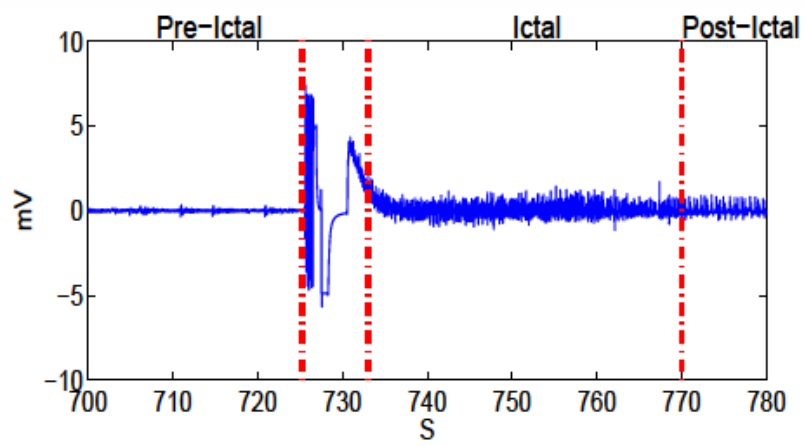

Figure 1: Wistar rat $E C o G$, evolution of an elicited seizure stage five, showing the Pre-Ictal, Ictal and Post-Ictal states. Intracranially recorded at the piriform cortex.

Seizures were recorded from 3 different Wistar rats, from the first stimulus to the end of the seizure, some of them could last up to 3 minutes; from here on we refer to the Wistar rats as subjects. Subjects respond in different ways to stimuli, most of the times more than one stimulus is necessary to achieve stage five seizures. In Figure 1, an $E C o G$ from a seizure rated as stage 5 is shown, it is possible to see a whole seizure evolution, with the Pre-Ictal state ending at second 727 , followed by the Ictal state that is considered after the stimuli from second 733 up to second 770 , where the Post-Ictal state starts. The $E C o G$ database was created from the elicited seizures produced daily over the course of 8 to 10 days on each subject, the $E C o G$ were sampled at $256 \mathrm{~Hz}$ and bandwidth limited from $0.5 \mathrm{~Hz}$ to $100 \mathrm{~Hz}$.

\subsection{Adaptive decomposition}

According to Franaszczuk et al. (1998), seizure evolution is a dynamic process with signals composed of multiple frequency components, which show 
up at different times with diverse durations. Therefore, it is convenient to analyze the signals with a method that provides a description of the signal's frequency evolution over time, which allow us to identify the seizure states in a rapidly changing signal, as ECoG does (Lorenzo and Biey, 2000).

To find the frequency components from a signal it is possible to perform a successive approximation through a set of candidate functions. The $M Z M P$ decomposes any signal into a linear expansion of waveforms which are selected from a redundant set of functions, called a dictionary (Mallat and Zhang, 1993). Taking this into account, spikes and sharp waves could be decomposed over functions well concentrated in time and with high bandwidth, while rhythmic waves could be better represented by waveforms which have a narrow frequency bandwidth. According to Franaszczuk et al. (1998), the $M Z M P$ allows a time-frequency $(T-F)$ analysis of a signal's components whose localization in time and frequency vary widely. Therefore, using the $M Z M P$ on $E C o G$ may result on a detailed description that can be used to classify and identify the dynamics of epileptic seizure states.

The $M Z M P$ algorithm decompose a signal through $m$ approximations to the signal $f(t)$ using waveforms from a dictionary of functions $D=$ $\left\{g_{1}, g_{2}, \ldots, g_{r}\right\}$ where each $g_{n}(t) \in \mathbb{L}^{2}(\mathbb{R})$. The number of waveforms $r$ in $D$ must be initialized prior to the start of the decomposition process. The algorithm performs a scaling, translation and modulation of a single window function $g(t)$ from the dictionary. This generates a family of components called atoms, which are well located in time and frequency; the signal decomposition is approximated by 


$$
f=\sum_{n=1}^{m}\left\langle R^{n} f, g_{n}\right\rangle g_{n}+R^{m} f,
$$

where $\left\langle R^{n} f, g_{n}\right\rangle$ is the inner product of the residue $R^{n} f$ with atom $g_{n}$, and $R^{m} f$ is the residual vector after $m$ iterations. This expansion minimizes the error $\varepsilon$ after $M$ approximations,

$$
\varepsilon=\left\|f(t)-\sum_{i=1}^{M} \omega_{i} g_{\gamma_{i}}(t)\right\|,
$$

where $\omega_{i}$ are the weights of the chosen functions $g_{\gamma_{i}}$. The approximation process can be stopped by implementing any of four different criteria: a) the Mallat and Zhang (1993) criterion that is related to the first $m$ atoms that have a higher than average correlation with the residue $R^{m} f$; b) the ratio of the total explained energy by the signal's components already found; c) predefined number of iterations neglecting the explained energy; and d) the energy of the last atom added, the energy resolution criterion (Jouny et al., 2003). Considering that the seizure states are identified based on signal amplitude, here we decided to use the explained energy as the stop criterion. According to Mallat and Zhang (1993), the $M Z M P$ computes the signal's energy by a derived energy distribution, consisting in adding the Wigner distribution of the selected atoms $\left(W g_{n}(t, \omega)\right)$; therefore, the epoch's energy $\left(E_{E}\right)$ is approximated by

$$
E_{E}(t, \omega)=\sum_{n=1}^{m}\left|\left\langle R^{n} f, g_{n}\right\rangle\right|^{2} W g_{n}(t, \omega) .
$$

The $M Z M P$ algorithm has been considered by Jouny et al. (2003) as a robust algorithm, which provides information about the rhythmic and 
transient activity of brain signals, and has been recommended as an approach specially suitable for analysis of non-stationary signals (Niedemeyer and Lopes da Silava, 1993). According to Blinowska (2001), MZMP is capable of providing a high resolution and parametric description of all kinds of data structures, making it possible to describe brain signals in terms of standard parameters; such as amplitude and $T-F$ location (Durka, 2004). The set of functions used to initialize the dictionary are Fourier functions, Delta functions and Gabor functions (Mallat and Zhang, 1993; Durka, 2004; Jouny et al., 2010). These functions are presumed to be more adaptable to the $E C o G$ rhythmic behavior, spike, and spike-wave complex, respectively. Using an adapted version based on the source code of the Piotr Durka MZMP software implementation ${ }^{1}$, Figure 2 shows the time-frequency distribution ( $T$ - FD) of a test signal composed of sinusoids from four different frequencies with different duration and time location, with four high amplitude spikes. It is possible to see that rhythmic signals, as the sinusoids are, have an energy $T-F$ representation depicted as narrow ellipses stretched along the time axis whose longitude is proportional to the components' duration. The rhythmic components' energy is distributed through narrow bands around the central frequency; this example is at $5 \mathrm{~Hz}, 10 \mathrm{~Hz}, 20 \mathrm{~Hz}$ and $40 \mathrm{~Hz}$. The transitory behavior characterized by spikes is represented by lines or narrow ellipses parallel to the frequency axis, meaning that each spikes' energy is distributed through a wide frequency band in a very short period of time; as depicted at seconds $0.3,0.7,1.3,1.8$ and 1.1 , respectively.

\footnotetext{
${ }^{1}$ The source code can be downloaded from http://eeg.pl/mp
} 

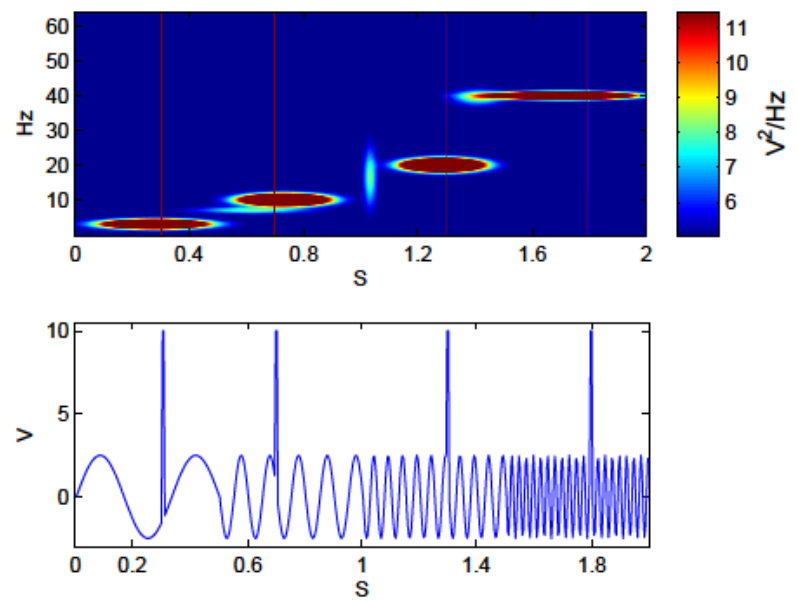

Figure 2: Signal decomposition by $M Z M P$ algorithm: the upper part shows the $T-F D$ of a signal, below of it the analyzed signal.

$E C o G$ decomposition produces a set of atoms, the size of which depends on the seizure evolution and signal length. The ratio of the number of atoms found $(m)$ to the $T-F$ window size is called the Gabor Atom Density which is considered by Jouny et al. (2004) a measure of a signal complexity, and is computed as

$$
G A D=\frac{m}{\Delta t \Delta f}=\frac{2 m}{N},
$$

where $\Delta t$ and $\Delta f$ are the $T-F$ window size and $N$ is the number of points in the signal.

According to John and Langley (1995), it is possible to assume that within each class the values of numeric attributes are normally distributed, allowing to represent the training-data distribution in terms of its mean and standard 
deviation. Our approach to identify the seizure states is to use a function that assigns an unknown sample to a class label, in this case we use the probabilistic Naive Bayes classifier which is considered an efficient classification algorithm (Zhang, 2005; Zhang and Jiang, 2008). Considering Theodoridis and Koutroumbas (2009), the classifier is constructed from a given set of training attributes, which assign an unknown sample $\mathbf{x}=\left[x_{1}, x_{2}, \ldots x_{l}\right]^{T}$, to the class

$$
\omega_{m}=\underset{\omega_{i}}{\operatorname{argmax}} \prod_{j=1}^{l} p\left(x_{j} \mid \omega_{i}\right), i=1,2, \ldots, M .
$$

Where $l$ is the number of features and $M$ the number of classes.

\subsection{Implementation}

In order to compute the $G A D$ and the $T-F$ distribution, we used our adapted version of the Piotr Durka $M Z M P$ software. The modifications consist on redirecting the output results to our analysis software, which does not compromise the signal approximation.

The $G A D$ computations were performed over the deep recording $E C o G$ from three subjects. Before processing, the $E C o G$ were digitally filtered in a bandwidth from $0.5 \mathrm{~Hz}$ to $60 \mathrm{~Hz}$. The ECoG were analyzed taking short segments of 2 seconds in length (Blanco et al., 1995; Stastny et al., 2001; Harikumar and Narayanan, 2003; Sotelo et al., 2007), called epochs. It is desirable that an online EES classification system has to come to a decision in a time interval less than the epoch's duration. These lead us to the need to identify the seizure states evaluating a small difference in the amount of information between nearby epochs. In view of that we propose to take 
the epochs with no overlap, and an overlap of $50 \%$ and $90 \%$. This means that an online system has to wait for a decision $100 \%, 50 \%$ and $10 \%$ of the duration of the epoch respectively. In order to find out the the best $G A D$ definition among seizure states with the less computing time, one experiment was setup for a decomposition level of $90 \%$ and the dictionary of functions was set to $70 \mathrm{~K}, 80 \mathrm{~K}, 90 \mathrm{~K}$ and $100 \mathrm{~K}$ atoms ${ }^{2}$. On a second experiment, decomposition level was set to $50 \%$ and $70 \%$ and dictionary set to $80 \mathrm{~K}$ atoms. The $E C o G$ were decomposed into their atoms, then the $G A D$ was computed using Equation (4).

Figure 3(a) shows a $T-F$ distribution of the Pre-Ictal state from subject 1 where signal decomposition was achieved using $80 \mathrm{~K}$ atoms, explaining $50 \%$ of the energy using ECoG segments of 28 seconds, the data for the GAD plot was computed under the same conditions, over 2 second epochs every 0.1 seconds. Looking at seconds 7, 13.9 and 25.8 it is possible to see three wavecomplexes and their components. The lobes stretched over the time axis are narrow-band components that explain the rhythmic nature of the wave, and those stretched over the frequency axis and of short duration are wide-band components that describe the transitory behavior of the spike. Average signal amplitude is very low, and atoms are concentrated below $10 \mathrm{~Hz}$. Figure 3(b) shows a $T-F D$ for the Ictal state from the same seizure, it is represented by Gabor functions describing the rhythmic nature of the Ictal state. It is evident that the $E C o G$ ' amplitude is increasing as the state progresses. In Figure 3(c) the Post-Ictal state from the same seizure is depicted, the

\footnotetext{
${ }^{2}$ Suffix K denotes thousands.
} 
$E C o G$ amplitude is actually decreasing; however, the atoms show a mainly long lasting rhythmic behavior. The continuous plot represents the GAD's evolution along the seizure states.

Since the $M Z M P$ is a greedy algorithm, it matches the coarser signal's structures with high energy atoms in the first iterations, the remaining finer structures are fitted with atoms with smaller influence in overall energy and $T-F$ distribution than the first atoms found. At a higher decomposition level for the same signal the very first atoms will be the same; however, more small energy atoms may be found, consequently the $G A D$ 's value may increase. From the same seizure of subject 1 , Figure $4(\mathrm{a}-\mathrm{c})$ show the $T$ - FDs with a decomposition level of $90 \%$ and a $100 \mathrm{~K}$ atoms dictionary. It is possible to observe that the GAD's magnitude increases, and the difference among the seizure states become more evident. Bringing to attention that the $G A D$ values are smaller than those reported by Jouny et al. (2003), which might be attributed to fact that the analyzed signals are from the seizure foci, where the detected potential is attributed to the influence of the nearby cells only, generating a less complex signal.

Figure 5 shows superimposed plots of the $G A D$ from four non-consecutive seizure episodes taken on different days from subject 1. In Figure 5(a) the energy level decomposition was set to $50 \%$ with a dictionary of $80 \mathrm{~K}$ atoms. The Pre-Ictal state begins from the first sample up to sample 160, where the Ictal state starts, the Post-Ictal state starts near sample 490. Though the seizures are not fully synchronized it is possible to observe that their respective $G A D$ changes its magnitude approximately the same way the seizures evolve. As the decomposition level and number of atoms in the dictionary are 

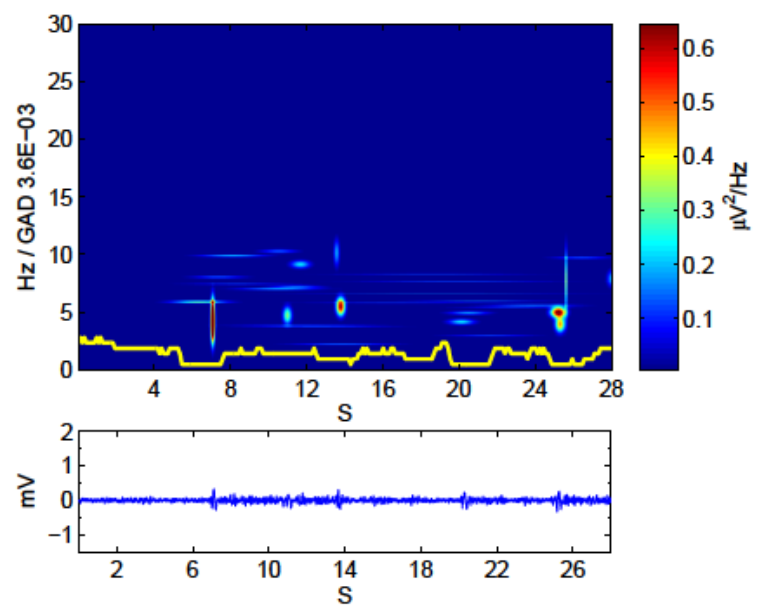

(a)
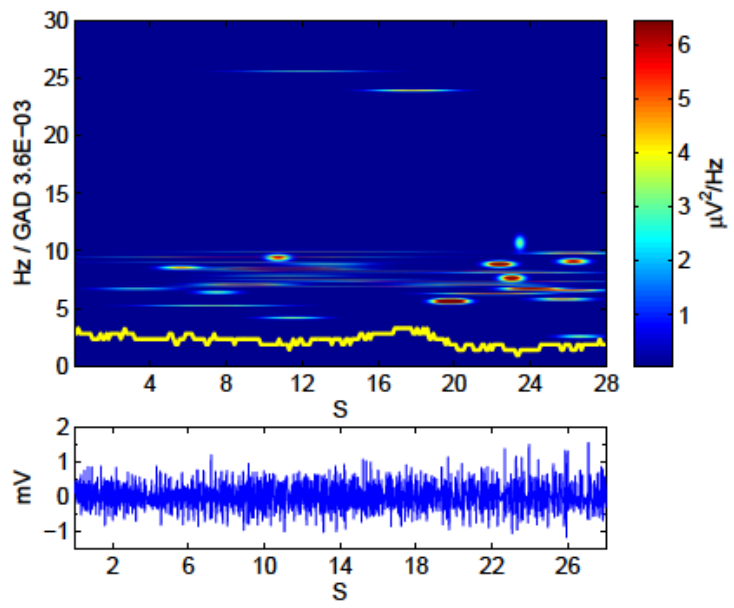

(b)
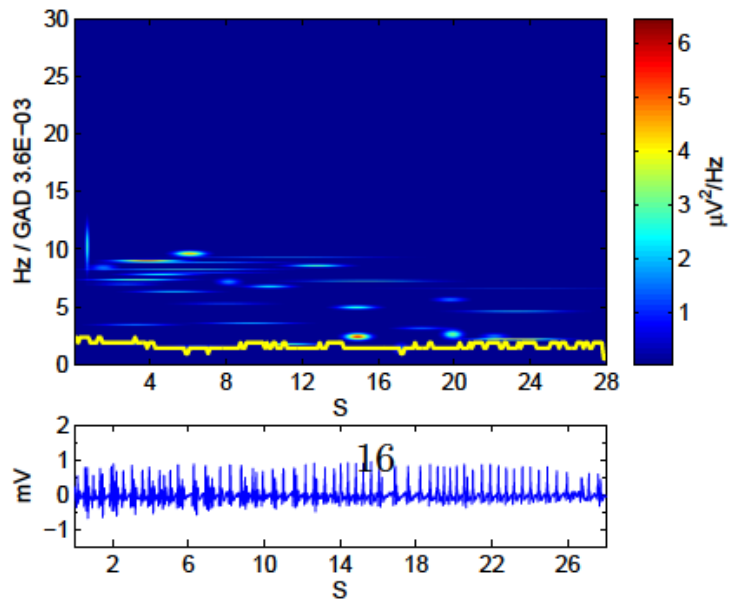

(c)

Figure 3: $T-F D$ from evolution of: (a) Pre-Ictal state, (b) Ictal state and (c) Post-Ictal state. Decomposition level of $50 \%$, with a dictionary of $80 \mathrm{~K}$ atoms. 


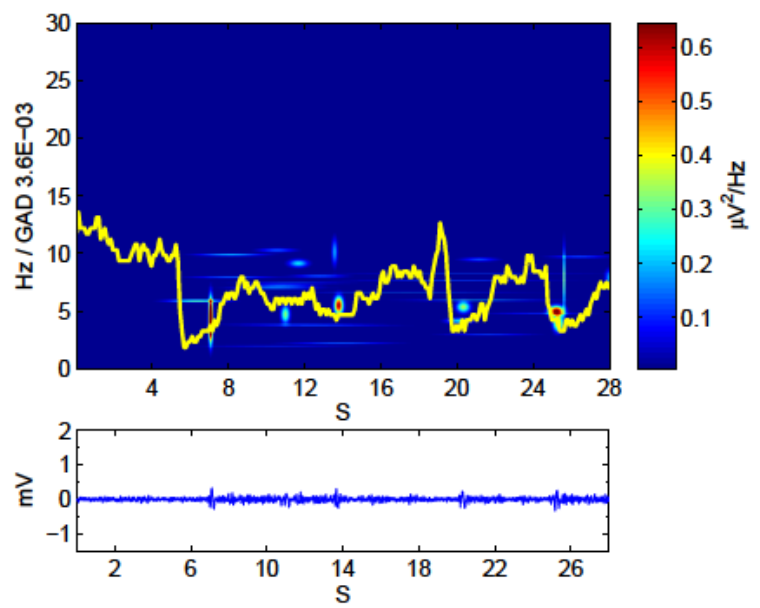

(a)
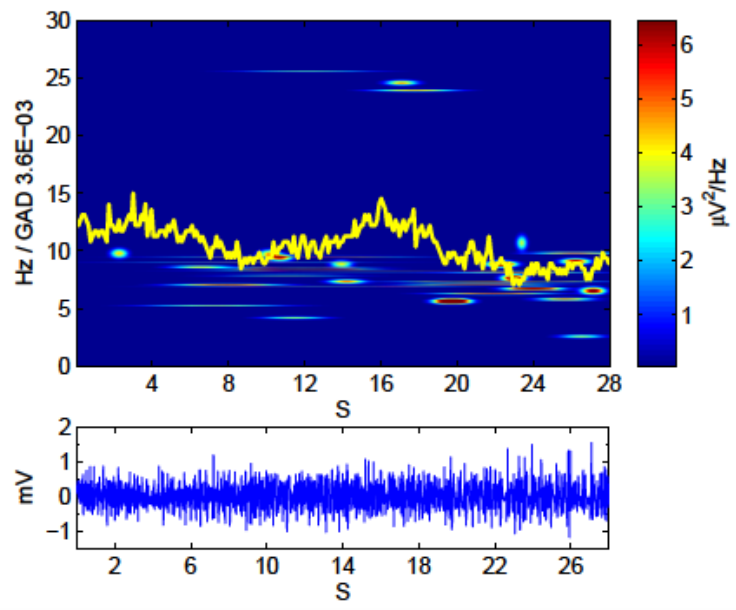

(b)
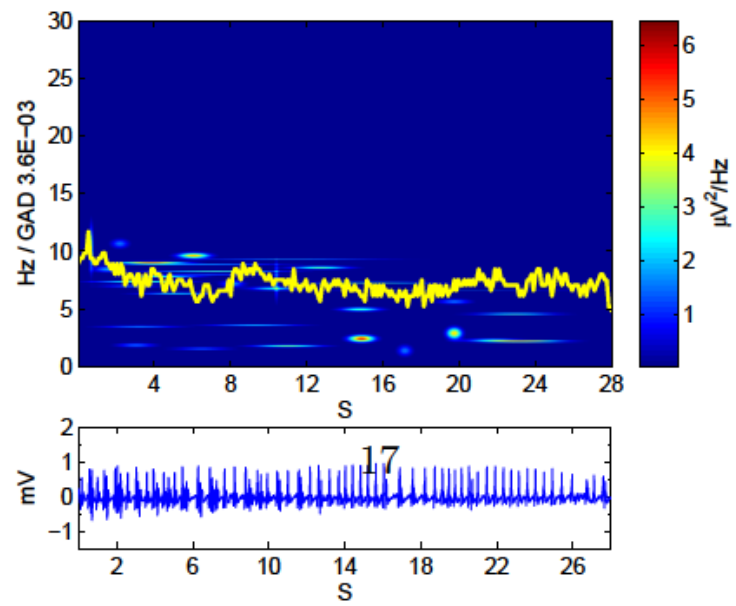

(c)

Figure 4: $T-F D$ from evolution of: (a) Pre-Ictal state, (b) Ictal state and(c) Post-Ictal state. Decomposition level of 90\%, with a dictionary of $100 \mathrm{~K}$ atoms. 
increased, the $G A D$ tendency is more evident, as is depicted in Figure 5(b) where the same seizure samples were decomposed at $90 \%$ of the energy using a dictionary of $100 \mathrm{~K}$ atoms.

Seizures from the same type of epilepsy share symptoms and the ECoG from different individuals share a similar morphology but exhibit different signal patterns (Franaszczuk et al., 1998), this is evident in the ECoG plots in Figure 6(a-b) belonging to subject 2 and subject 3 during a stage five elicited seizure. Further, in Figure 6(c-d) the respective $G A D$ from seizures episodes of non-consecutive days are plotted, and it is also possible to observe that the $G A D$ behaves differently between each subject.

\section{Results and discussion}

Jouny et al. (2004) suggest that the $G A D$ reflects the $E C o G$ dynamics and it changes as the seizure evolves; such a behavior can be observed clearly in Figure 6. Taking this into account, we use the $G A D$ as an indicator for the seizure state using small ECoG segments. The training data selection is based in the assumption that an online classifier must be trained efficiently, using the least amount of data as possible. Hence, in our tested scenario the training process only uses information from a single recording day. Furthermore, for a more realistic testing scenario, the classifier is tested on signals from non-consecutive days, to reduce any possible correlation between the training and testing signals. Therefore, three training data-sets were arranged using seizures from one, two and three different non-consecutive days. As the epoch length is already fixed, the amount of epochs in each state depends on the overlap and state duration. The total number of epochs in the 


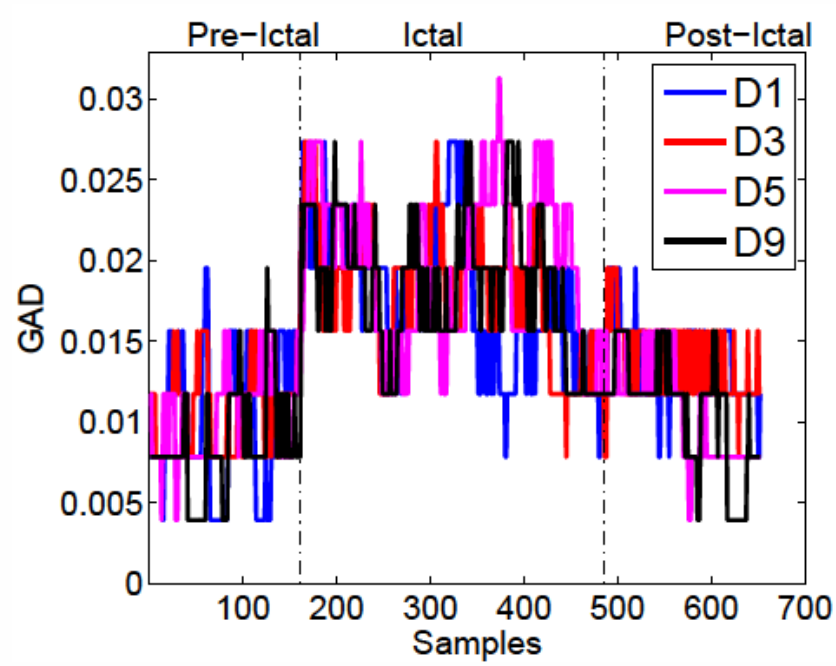

(a)

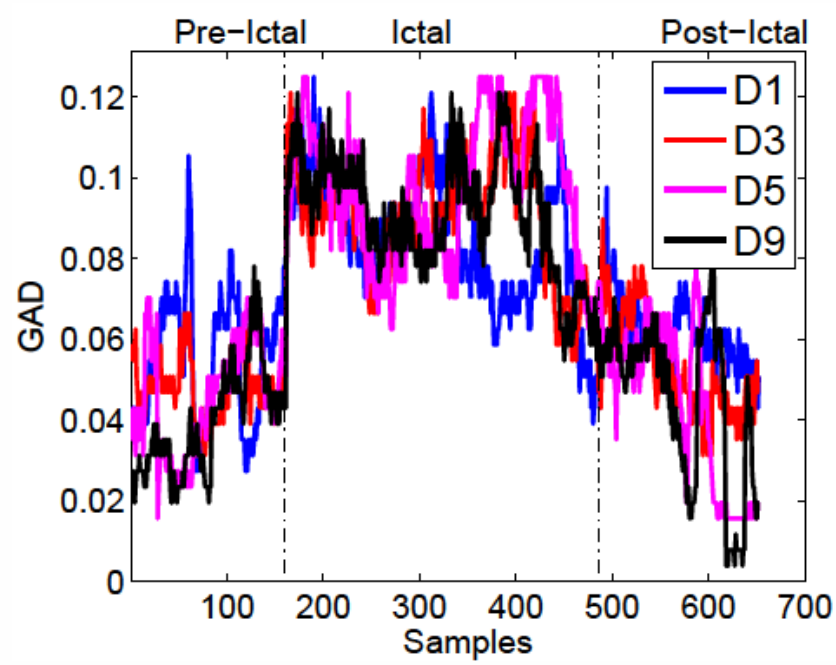

(b)

Figure 5: GAD plots from 4 different seizures, from days 1, 3, 5 and 9 of subject 1: (a) decomposition level of $50 \%$ and $80 \mathrm{~K}$ atoms, (b) decomposition level of $90 \%$ and $100 \mathrm{~K}$ atoms. 


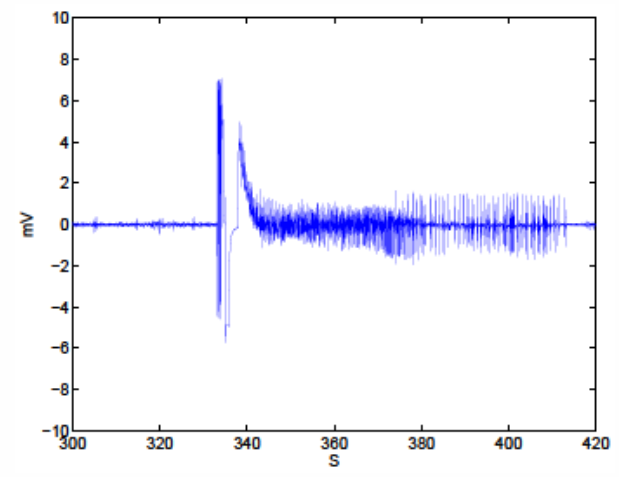

(a)

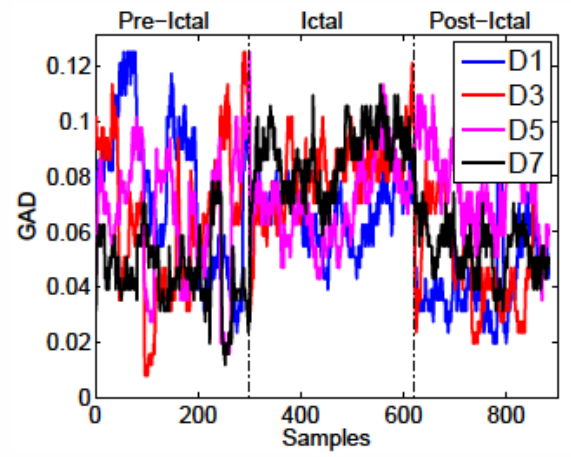

(c)

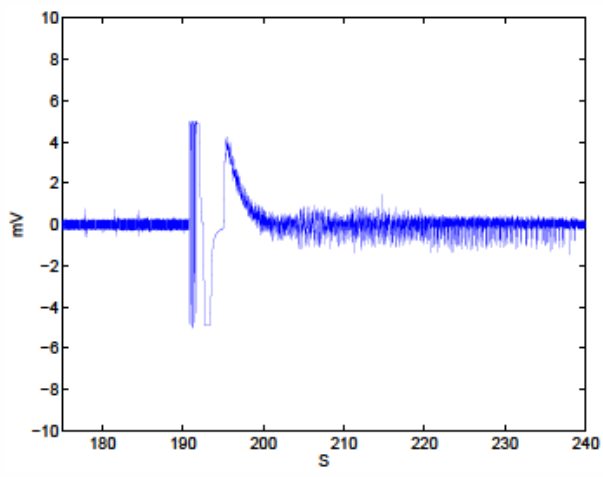

(b)

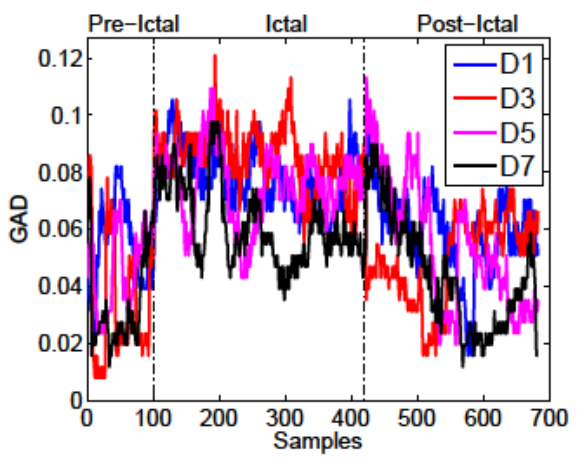

(d)

Figure 6: $E C o G$ plot from one seizure episode: (a) subject 2, and (b) subject 3. $G A D$ plot from four non-consecutive days: (c) for subject 2 , and (d) for subject 3 . In both cases decomposition level was set to $90 \%$, with a dictionary of $100 \mathrm{~K}$ atoms. 


\begin{tabular}{|c|c|c|c|c|c|}
\hline Overlap & Epochs & Overlap & Epochs & Overlap & Epochs \\
\hline Non & 48 & Non & 96 & Non & 144 \\
\hline $50 \%$ & 93 & $50 \%$ & 186 & $50 \%$ & 279 \\
\hline $90 \%$ & 453 & $90 \%$ & 906 & $90 \%$ & 1359 \\
\hline
\end{tabular}

(a)

(b)

(c)

Table 1: Number of epochs included as training-data, using seizures from: (a) one day, (b) two non-consecutive days, and (c) three non-consecutive days.

training data-sets are shown in Table 1. GAD was computed using a $100 \mathrm{~K}$ atoms dictionary and energy explanation of $90 \%$, considering that the best $G A D$ tendency was obtained within these values.

In order to build the Naive Bayes classifier we use the three different proposed classes, Pre-Ictal, Ictal and Post-Ictal, and two observed attributes: $G A D$ and $E_{E}$, which are assume to be normally distributed (John and Langley, 1995). The normalized $G A D$ member functions of the proposed classes are shown in Figure 7, from left to right the columns show plots using epochs from one, two and three days. Figure $7(\mathrm{a}-\mathrm{c})$ depicts distributions using non-overlapped epochs, Figure 7(d - f) using epochs with a 50\% overlap, and Figure $7(\mathrm{~g}-\mathrm{i})$ using epochs with a $90 \%$ overlap. It is possible to observe that $G A D$ from the Ictal state are clearly separated from the other two states, while Pre-Ictal and Post-Ictal are overlapped. The GAD from the Post-Ictal state is concentrated in a narrow band, making it difficult to discriminate among both states. This overlap is attributable to the fact that the $E C o G$ 
from these two states share a similar transitory behavior. However, as the epoch overlap increases as well as the number of seizures used as training data, the overlap between the Pre-Ictal and Post-Ictal states decreases a bit, but it is still not a significant difference which could allow to clearly discriminating between them. Therefore, a second attribute is required in conjunction with $G A D$ to avoid this ambiguity, we proposed the $E_{E}$.

For the second observed attribute, the normalized member functions are shown in Figure 8, from left to right the columns show plots using epochs from one, two and three days. Figure $8(\mathrm{a}-\mathrm{c})$ depicts distribution using non-overlapped epochs, Figure 8(d - f) using epochs with a 50\% overlap, and Figure $8(\mathrm{~g}-\mathrm{i})$ using epochs with a $90 \%$ overlap. It is possible to observe that $E_{E}$ from the different states is clearly identifiable, making it easy to discriminate among the three states. It is also possible to observe that the $E_{E}$ from the Ictal state has the highest energy level and the widest distribution, this energy distribution magnitude is followed by the Post-Ictal state.

Considering the energy decomposition of $90 \%$ and a $100 \mathrm{~K}$ atom dictionary, epochs with $90 \%$ and using seizures from 3 days gives the best $G A D$ and energy intervals to discriminates among the three seizure states. Figure 9 shows the $G A D$ and the $E_{E}$ distributions for the other two subjects. Figure $9(\mathrm{a}-\mathrm{b})$ for subject 2 ; Figure $9(\mathrm{c}-\mathrm{d})$ for subject 3 . The figures confirm that the $E C o G$ from different subjects may exhibit different dynamics, the $G A D$ intervals from these subjects have different means and ranges, however the $G A D$ in general lies within similar boundaries. In addition, this makes it difficult to classify seizure states from one subject with a classifier trained with seizures from another. 


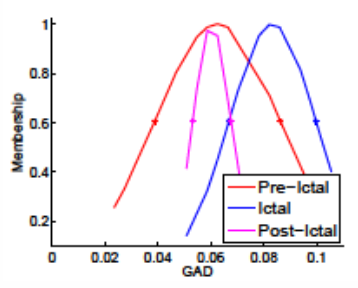

(a)

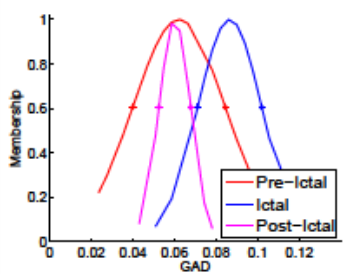

(d)

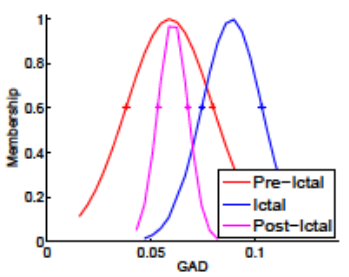

(g)

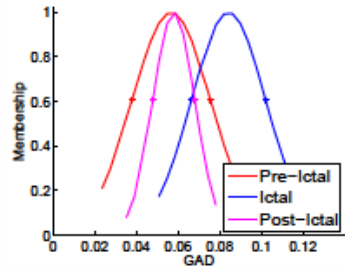

(b)

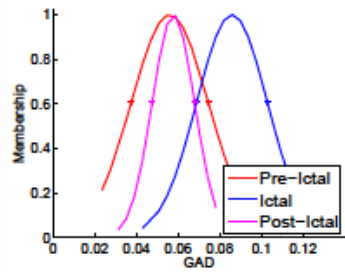

(e)

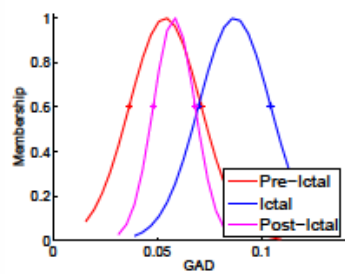

(h)

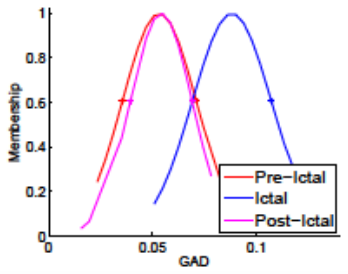

(c)

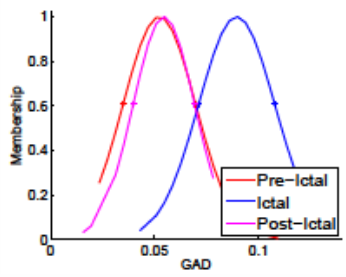

(f)

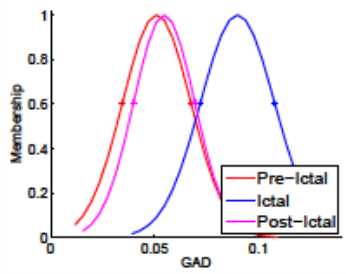

(i)

Figure 7: $G A D$ normalized member functions plots for the subject 1: (a c) non-overlapped epochs. (d - f) epochs with a 50\% overlap. (g - i) epochs with a $90 \%$ overlap. In the first column using epoch from the first day, in the second column using epoch from two non-consecutive days, and in third column using epochs from three non-consecutive days. 


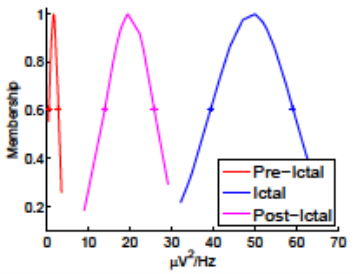

(a)

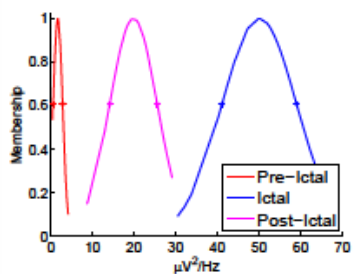

(d)

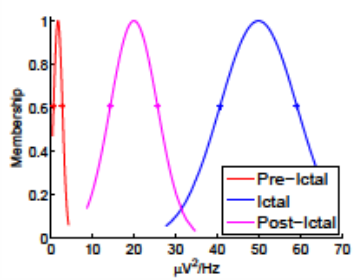

(g)

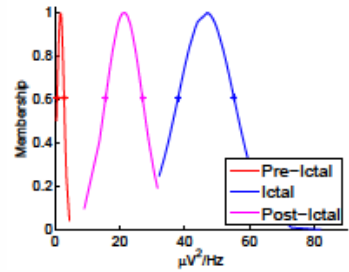

(b)

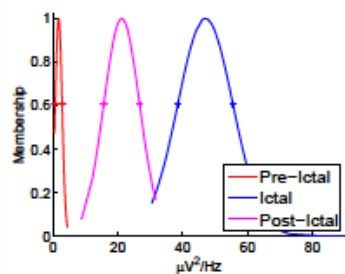

(e)

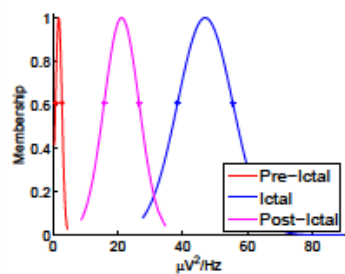

(h)

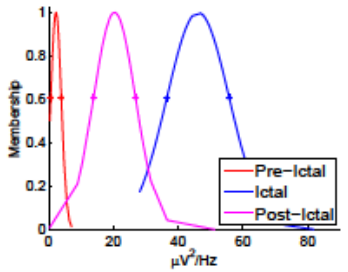

(c)

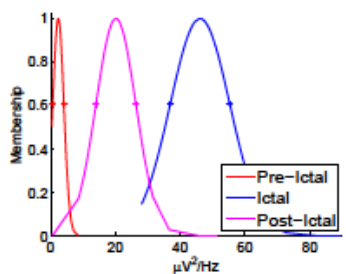

(f)

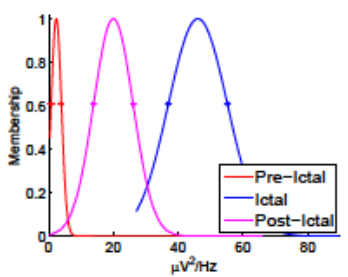

(i)

Figure 8: $E_{E}$ normalized member functions plots for subject 1: (a - c) nonoverlapped epochs. (d - f) epochs with a $50 \%$ overlap. (g - i) epochs with a $90 \%$ overlap. In the first column using epoch from the first day, in the second column using epoch from two non-consecutive days, and in third column using epochs from three non-consecutive days. 


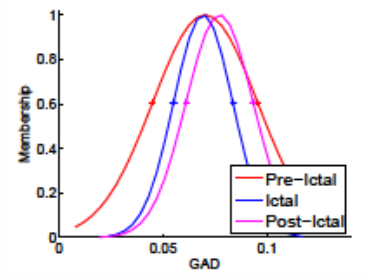

(a)

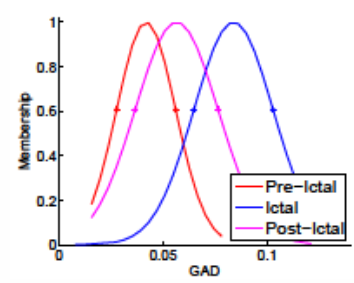

(c)

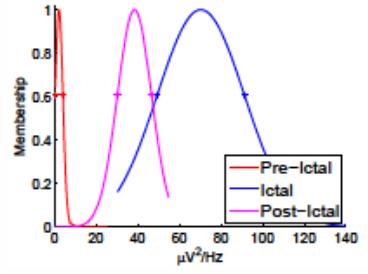

(b)

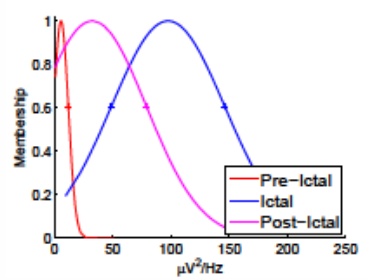

(d)

Figure 9: $G A D$ and $E_{E}$ normalized distribution plots for: (a - b) subject 2, (c - d) subject 3. Energy decomposition level of $90 \%$ and a dictionary with $100 \mathrm{~K}$ atoms, epochs with a $90 \%$ overlap and 3 days seizures. 
Taking in account that using both of the proposed attributes together should be suitable to discriminate the seizure states; skipping the seizures used to train, another set of epochs picked from different states were used to test the learned models using both features. The classification was performed over the corresponding trained classifier for each subject. After classification, results were evaluated according with the diagnostic test method (Altman, 2000). Confusion matrixes are shown in Table 2, where each row shows the classifications result for each state, the second column is the total number of epochs from each state, the third column are the number of true positives $(T P)$, the fourth column shows the number of false positives $(F P)$, the fifth column the number of true negatives $(T N)$, and in the sixth column the number of false negatives $(F N)$.

Typical classification results on epochs from a seizure of a single day are shown in Table 2(a) for subject 1 using 453 epochs, Table 2(b) for subject 2 using 424 epochs; and Table 2(c) for subject 3 using 704 epochs. Table 3 shows the classification results in epochs from seizures from 8 days, in a couple of those days there were only Pre-Ictal state, the classification result are shown in Table 3(a) for subject 1 using 5047 epochs, Table 3(b) for subject 2 using 5823 epochs, and Table 3(c) for subject 3 using 3786 epochs.

Table 4 shows the performance indexes computed for the classification results from Table 3. Sensitivity $(S E)$ is an indicator of how efficiently the features of observed attributes can identify a particular state. On the other hand, specificity $(S P)$ values characterize how efficiently the method rejects the features which are not from a particular state. It is possible to observe the Ictal state has a high $S E$ score on the three subjects, attributed to 


\begin{tabular}{lccccc}
\hline state & Total & TP & FP & TN & FN \\
\hline Pre-Ictal & 151 & 151 & 0 & 302 & 0 \\
Ictal & 171 & 168 & 0 & 282 & 3 \\
Post-Ictal & 131 & 131 & 3 & 319 & 0 \\
\hline
\end{tabular}

(a)

\begin{tabular}{lccccc}
\hline state & Total & TP & FP & TN & FN \\
\hline Pre-Ictal & 151 & 151 & 0 & 273 & 0 \\
Ictal & 142 & 142 & 131 & 151 & 0 \\
Post-Ictal & 131 & 0 & 0 & 293 & 131 \\
\hline
\end{tabular}

(b)

\begin{tabular}{lccccc}
\hline state & Total & TP & FP & TN & FN \\
\hline Pre-Ictal & 151 & 106 & 116 & 437 & 45 \\
Ictal & 422 & 394 & 7 & 275 & 28 \\
Post-Ictal & 131 & 18 & 63 & 510 & 113 \\
\hline
\end{tabular}

(c)

Table 2: Classification results on a seizure from a single day for: (a) subject 1; (b) subject 2; and (c) subject 3 . 


\begin{tabular}{lccccc}
\hline state & Total & TP & FP & TN & FN \\
\hline Pre-Ictal & 2484 & 2432 & 491 & 2072 & 52 \\
Ictal & 1558 & 1283 & 33 & 3456 & 275 \\
Post-Ictal & 1005 & 614 & 194 & 3848 & 391 \\
\hline
\end{tabular}

(a)

\begin{tabular}{lccccc}
\hline state & Total & TP & FP & TN & FN \\
\hline Pre-Ictal & 2701 & 2381 & 409 & 2713 & 320 \\
Ictal & 1943 & 1874 & 1158 & 2722 & 69 \\
Post-Ictal & 1179 & 0 & 1 & 4643 & 1179 \\
\hline
\end{tabular}

(b)

\begin{tabular}{lccccc}
\hline state & Total & TP & FP & TN & FN \\
\hline Pre-Ictal & 1303 & 612 & 477 & 2006 & 691 \\
Ictal & 1590 & 1313 & 477 & 1719 & 277 \\
Post-Ictal & 893 & 239 & 668 & 2225 & 654 \\
\hline
\end{tabular}

(c)

Table 3: Classification results using seizures from several days to test the classifier, for: (a) subject 1. (b) subject 2. (c) subject 3 . 
the fact that Ictal intervals show a significant difference compared with the other two states. After the Ictal score, the Pre-Ictal, and the Post-Ictal show a progressively lower performance, attributable to the fact that their $G A D$ intervals are inside the Pre-Ictal's range, causing false negative classification. For instance in the case of subject 2 the classification error is $100 \%$ for the Post-Ictal state.

Figure 10 shows the receiver operating characteristics curves $(R O C)$ of the seizure state classification for the three subjects. In most of the cases the $E_{E^{-}} R O C$ s are near to point $(0,1)$, suggesting that $E_{E}$ is a strong evidence for the presence of a seizure state (Fawcett, 2006), making positive identification. On the other hand, $G A D-R O C$ in some cases are near to the diagonal while still having good classification performance. The closer to the left-upper corner the better the classification performance (Metz, 1978).

The probability that a classifier correctly identifies a true positive case is describe by the area under the curve $(A U C)$ (Fawcett, 2006), Table 5 shows the $A U C \mathrm{~s}$ from the $G A D-R O C$ and $E_{E^{-}} R O C$ s for each of the subjects.

\section{Conclusion}

This paper presents a method to identify the three main states of a seizure evolution. The proposal is based on the classification of attributes extracted from short ECoG segments. The experimental results suggest that the seizure states are actually revealed by the signal's dynamic as the $G A D$ plots show, inferring that it is possible to classify seizure states using this feature. However, their ranges are not separated enough to have a totally clear classification, this overlapping can causes multiple false positive or false 


\begin{tabular}{lccccc}
\hline state & SE & SP & \%Error & TPF & FPF \\
\hline Pre-Ictal & 0.979 & 0.808 & 2.0 & 0.979 & 0.191 \\
Ictal & 0.823 & 0.990 & 17.6 & 0.823 & 0.009 \\
Post-Ictal & 0.610 & 0.952 & 38.9 & 0.610 & 0.047 \\
\hline
\end{tabular}

(a)

\begin{tabular}{lccccc}
\hline state & SE & SP & \%Error & TPF & FPF \\
\hline Pre-Ictal & 0.881 & 0.868 & 11.8 & 0.881 & 0.131 \\
Ictal & 0.964 & 0.701 & 3.5 & 0.964 & 0.298 \\
Post-Ictal & 0 & 0.999 & 100 & 0 & 0.000 \\
\hline
\end{tabular}

(b)

\begin{tabular}{lccccc}
\hline state & SE & SP & \%Error & TPF & FPF \\
\hline Pre-Ictal & 0.469 & 0.807 & 53.0 & 0.469 & 0.192 \\
Ictal & 0.825 & 0.782 & 17.4 & 0.825 & 0.217 \\
Post-Ictal & 0.267 & 0.769 & 73.2 & 0.267 & 0.230 \\
\hline
\end{tabular}

(c)

Table 4: Performance indexes from the classification results shown in Table 3, for: (a) subject 1; (b) subject 2; and (c) subject 3. 


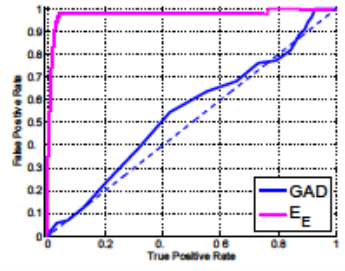

(a)

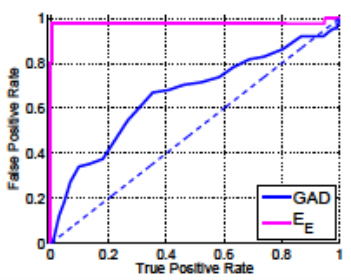

(d)

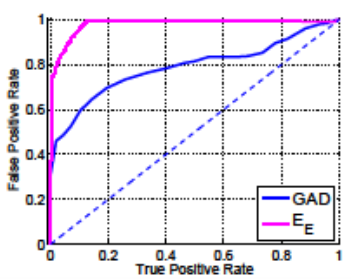

(g)

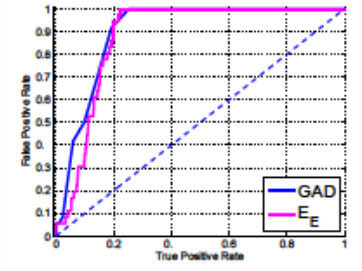

(b)

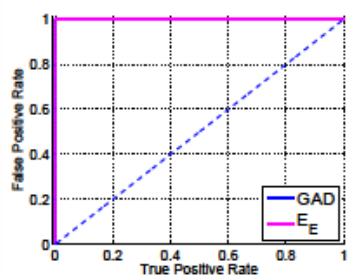

(e)

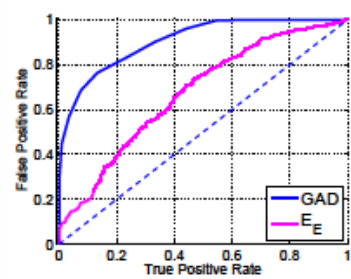

(h)

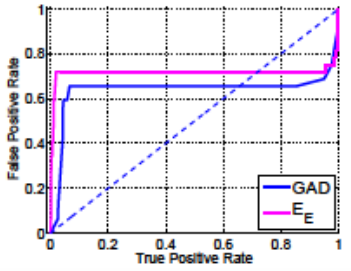

(c)

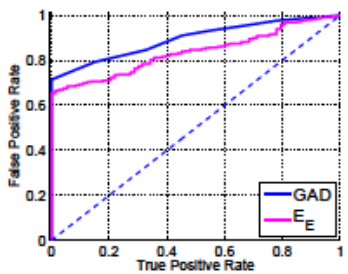

(f)

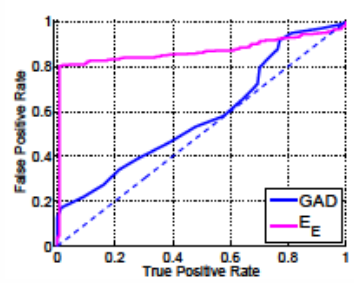

(i)

Figure 10: $R O C$ plots for Pre-Ictal, Ictal and Post-Ictal states for: $(\mathrm{a}-\mathrm{c})$ subject $1 ;(\mathrm{d}-\mathrm{f})$ subject 2 , and $(\mathrm{g}-\mathrm{i})$ subject 3 . 


\begin{tabular}{lcc}
\hline state & AUC-GAD & AUC-Energy \\
\hline Pre-Ictal & 0.543 & 0.975 \\
Ictal & 0.900 & 0.881 \\
Post-Ictal & 0.631 & 0.717 \\
\hline
\end{tabular}

(a)

\begin{tabular}{lcc}
\hline state & AUC-GAD & AUC-Energy \\
\hline Pre-Ictal & 0.661 & 0.978 \\
Ictal & 0.999 & 0.999 \\
Post-Ictal & 0.895 & 0.836 \\
\hline
\end{tabular}

(b)

\begin{tabular}{lcc}
\hline state & AUC-GAD & AUC-Energy \\
\hline Pre-Ictal & 0.789 & 0.978 \\
Ictal & 0.907 & 0.673 \\
Post-Ictal & 0.581 & 0.870 \\
\hline
\end{tabular}

(c)

Table 5: Area under the curve for the ROC curves from Figure 10 for: (a) subject 1, (b) subject 2, and (c) subject 3 . 
negative when only $G A D$ is used. Nonetheless, the $E_{E}$ has clearer intervals, and combining both features produces an improved classifier with accuracy between $55 \%$ to $79 \%$ for the Pre-Ictal state and between $90 \%$ to $99 \%$ for the Ictal state. These results shows considerable improvement when compared with a previous classification method, where average specificity was $12 \%$ (Sotelo et al., 2007), and similar accuracy compared with respect to the 88.9\% reported in (Sotelo et al., 2012) and the 99\% reported in (Sotelo et al., 2013) using similar data. These results are promising considering the performance reported from similar works using different techniques, where Buteneers et al. (2013) reports $2.8 \%$ average error rate of seizure detection and the $99.125 \%$ accuracy identifying seizure and non-seizure activity in humans reported by $\mathrm{Fu}$ et al. (2014). If we consider that during the evolution of an epileptic seizure the Pre-Ictal state always precede the Ictal state, and the Post-Ictal state unlikely precedes the Pre-Ictal, then these numbers are promising in the online classification of seizure states. Furthermore, it is shown that small changes in the signal, overlap of $90 \%$, allow to identify the seizure state.

It is important to remark the fact that epileptic seizures share $E C o G$ morphology among individuals, nevertheless, they do not share ECoG's dynamic characteristics. This is an important matter when the goal is to find an automatic seizure state classification method, since a parameter which works fine for one individual might not work properly for another. Suggesting that the classification method must be tuned according to the particular seizure characteristics from a particular individual, and probably to the particular epilepsy type. 
Nevertheless, more work has to be done to get a refined classification method based on $E C o G$ dynamics estimated by means of $G A D$. Considering that the $G A D$ is computed on Fourier, Delta, and Gabor functions; and the fact that the Pre-Ictal and Post-Ictal states are from a transitory nature. Hence the atom density of each of these three components might be used as observed attributes in the classifier to discriminate these two behaviorally similar states.

\section{Acknowledgement}

Thanks are extended to the Hospital Universitario de Valencia, for sharing their signal database and to Francisco Sancho for his support. Finally, thanks are given to Dr. Luis N. Coria for his support and the proofreading support.

Partial funding for this work was provided by CONACYT Basic Science Research Project No. 178323, DGEST (Mexico) Research Projects No. 5149.13-P, 5414.14-P and TIJ-ING-2012-110, and IRSES project ACoBSEC financed by the European Commission.

\section{References}

Altman DG. Diagnostic Test. 1st ed. Statistics with Confidence. London: BMJ Publishing Group, 2000.

Blanco S, Quiroga RQ, Rosso OA, Kochen S. Time-frequency analysis of electroencephalogram series. Phys Rev E 1995;51:2624-31.

Blinowska K. Unbiased high resolution method of eeg analysis in timefrequency space. Acta Neurobiol Exp 2001;61:157-74. 
Buteneers P, Verstraeten D, Nieuwenhuyse BV, Stroobandt D, Raedt R, Vonck K, Boon P, Schrauwen B. Real-time detection of epileptic seizures in animal models using reservoir computing. Epilepsy Research 2013;103(23):124-34.

Cockerell O. Epilepsy, Current Concepts. 2nd ed. Current Medical Literature LTD, 2003.

Curtis MD, Avanzini G. Interictal spikes in focal epileptogenesis. Progress in Neurobiology 2001;63:541-67.

Durka PJ. Adaptive time-frequency parametrization of epileptic spikes. Physical Review E (Statistical, Nonlinear, and Soft Matter Physics) 2004;69(5).

Fawcett T. An introduction to roc analysis. Pattern Recognition Letters 2006;27(8):861 -74.

Franaszczuk PJ, Bergey G, Durka P, Eisenberg H. Time-frequency analysis using the matching pursuit algorithm applied to seizures originating from the mesial temporal lobe. ElectroencephClinNeurophysiol $1998 ; 106(6): 513-21$.

Freeman FG, Jarvis MF. The effect of interstimulation interval on the assessment and stability of kindled seizure thresholds. Brain Research Bulletin 1981;7(6):629-33.

Fu K, Qu J, Chai Y, Dong Y. Classification of seizure based on the timefrequency image of eeg signals using hht and svm. Biomedical Signal Processing and Control 2014;13(0):15 - 22 . 
Gallego JM, Sancho FJ, Vidueira S, Ortiz L, Gómez-Pinedo U, Barcia JA. Injection of embryonic median ganglionic eminence cells or fibroblasts within the amygdala in rats kindled from the piriform cortex. Seizure 2010;19(8):461-6.

Goddard GV. The kindling model of epilepsy. Trends in Neurosciences $1983 ; 6(0): 275-9$.

Harikumar R, Narayanan B. Fuzzy techniques for classification of epilepsy risk level from eeg signals. In: TENCON 2003. Conference on Convergent Technologies for the Asia-Pacific Region. volume 1; 2003. p. 209-13.

Iasemidis L, Shiau DS, Chaovalitwongse W, Sackellares J, Pardalos P, Principe J, Carney P, Prasad A, Veeramani B, Tsakalis K. Adaptive epileptic seizure prediction system. Biomedical Engineering, IEEE Transactions on $2003 ; 50(5): 616-27$.

John GH, Langley P. Estimating continuous distributions in bayesian classifiers. In: Proceedings of the Eleventh Conference on Uncertainty in Artificial Intelligence. San Francisco, CA, USA: Morgan Kaufmann Publishers Inc.; UAI'95; 1995. p. 338-45.

Jouny CC, Bergey GK, Franaszczuk PJ. Partial seizures are associated with early increases in signal complexity. Clinical Neurophysiology 2010;121(1):7 - 13 .

Jouny CC, Franaszczuk P, Adamolekun B, Bergey G. Gabor atom density as a measure of seizure complexity 2004;1:310 -2. 
Jouny CC, Franaszczuk PJ, Bergey GK. Characterization of epileptic seizure dynamics using gabor atom density. Clinical Neurophysiology $2003 ; 114(3): 426-37$.

Kohsaka S, Mizukami S, Kohsaka M, Shiraishi H, Kobayashi K. Widespread activation of the brainstem preceding the recruiting rhythm in human epilepsies. Neurosciences 2002;115(3):697-706.

López-Cuevas A, Castillo-Toledo B, Medina-Ceja L, Ventura-Mejía C, PardoPeña K. An algorithm for on-line detection of high frequency oscillations related to epilepsy. Computer Methods and Programs in Biomedicine 2013;110(3):354-60.

Lorenzo G, Biey M. Analysis of chaotic signals in the time-frequency plane. Journal of signal processing 2000;4(1):29-36.

Mallat S, Zhang Z. Matching pursuits with time-frequency dictionaries. Signal Processing, IEEE Transactions on 1993;41(12):3397 -415.

McIntyre DC, Gilby KL. Kindling as a model of human epilepsy. The Canadian Journal Of Neurological Sciences Le Journal Canadien Des Sciences Neurologiques 2009;36 Suppl 2:S33 -5.

Metz CE. Basic principles of roc analysis. Seminars in Nuclear Medicine 1978;8(4):283-98.

Morimoto K, Fahnestock M, Racine RJ. Kindling and status epilepticus models of epilepsy: rewiring the brain. Progress in Neurobiology 2004;73(1):1 $-60$. 
Nair SP, Shiau DS, Principe JC, Iasemidis LD, Pardalos PM, Norman WM, Carney PR, Kelly KM, Sackellares JC. An investigation of eeg dynamics in an animal model of temporal lobe epilepsy using the maximum lyapunov exponent. Experimental Neurology 2009;216(1):115 -21.

Niedemeyer E, Lopes da Silava F. Electroencephalography:Basic Principles, Clinical Applications and Related Fields. 3rd ed. Williams\&Wilkins, 1993.

Niknazar M, Mousavi S, Motaghi S, Dehghani A, Vahdat BV, Shamsollahi M, Sayyah M, Noorbakhsh S. A unified approach for detection of induced epileptic seizures in rats using ecog signals. Epilepsy \& Behavior $2013 ; 27(2): 355-64$.

Racine RJ. Modification of seizure activitiy by electrical stimulation. ii motor seizure. Electroencephalography and Clinical Neurophysiology 1972;(32):281-94.

Rangayyan RM. Biomedical Signal Analysis: a case-study approach. IEEE Press Series on Biomedical Engineering. John Wiley \& Sons, Inc., 2002.

Roso O, Figliola A. Order/disorder in brain electrical activity. Revista Mexcana de Física 2004;50(2):149-55.

Sotelo A, Guijarro E, Coria LN, Trujillo L, Valle PA. Epilepsy ictal stage identification by $0-1$ test of chaos. In: Proceedings of the third IFAC CHAOS Conference. volume 1; 2012. p. 223 -8.

Sotelo A, Guijarro E, Trujillo L, Coria LN, Martínez Y. Identification of epilepsy stages from ecog using genetic programming classifiers. Computers in Biology and Medicine 2013;43(1):1713-23. 
Sotelo E, Guijarro E, Garcia M, Vazquez C. Epoch parameterization by gabor atom density in experimental epilepsy. In: Electrical and Electronics Engineering, 2007. ICEEE 2007. 4th International Conference on. 2007. p. $61-4$.

Stastny J, Sovka P, Stancak A. Eeg signal classification. In: Engineering in Medicine and Biology Society, 2001. Proceedings of the 23rd Annual International Conference of the IEEE. volume 2; 2001. p. 2020-2023 vol.2.

Theodoridis S, Koutroumbas K. Pattern recognition. 4th ed. Elsevier, 2009.

WHO . Atlas: Epilepsy Care in the World. World Healt Organizaion, 2005.

Williams W, Zaveri H, Sackellares J. Time-frequency analysis of electrophysiology signals in epilepsy. Engineering in Medicine and Biology Magazine, IEEE 1995;14(2):133-43.

Witee H, Iasemidis L, Litt B. Special issue on epileptic seizure prediction. IEEE transactions on biomedical engineering 2003;50(5):537-9.

Zaveri H, Williams W, Iasemidis L, Sackellares J. Time-frequency representation of electrocorticograms in temporal lobe epilepsy. Biomedical Engineering, IEEE Transactions on 1992;39(5):502 -9.

Zhang H. Exploring conditions for the optimality of naïve bayes. International Journal of Pattern Recognition \& Artificial Intelligence 2005;19(2):183-98.

Zhang H, Jiang S. Naive bayes for optimal ranking. Journal of Experimental \& Theoretical Artificial Intelligence 2008;20(2):79 - 93. 